\title{
Análise de custo dos materiais usados para realizar as técnicas anestésicas na cirurgia progiramada de extração de catarata por facoemulsificação
}

\author{
Costs oflocal anesthesia technique used in ophthalmology to perform cataract \\ surgeryby phacoemulsification
}

Jefferson Barreiro ${ }^{1}$

Telma Pereira Barreiro ${ }^{2}$

José Ricardo Carvalho Lima Rehder ${ }^{3}$

\section{RESUMO}

Objetivo: Comparar o custo das técnicas anestésicas locais usadas em oftalmologia (retrobulbar, peribulbar, subtenoniana e tópica) para realizar cirurgia de facoemulsificação. Métodos: Para análise de custo dos materiais usados para realizar os diferentes procedimentos anestésicos, adotamos as técnicas padronizadas pelo Setor de Catarata da Disciplina de Oftalmologia da Faculdade de Medicina do ABC. Para cada técnica foram tabulados os valores totais correspondentes aos materiais necessários para realizar os procedimentos anestésicos em questão. Todos os materiais utilizados foram adquiridos após a análise de preços de três fornecedores, tendo sido escolhido o de menor valor. Resultados: $O$ custo da anestesia tópica foi de 0,06 reais, da anestesia retrobulbar foi de 7,42 reais, da anestesia peribulbar foi de 24,96 reais e o da anestesia subtenoniana foi de 48,12 reais. Conclusões: 1. A anestesia tópica foi à técnica que apresentou o menor custo. 2. A anestesia retrobulbar apresenta custo mais baixo quando comparado a peribulbar e subtenoniana. 3. A anestesia subtenoniana apre-senta custo mais elevado quando comparada a outras técnicas utilizadas neste estudo.

Descritores: Custos de cuidados de saúde; Anestesia local/economia; Extração de catarata/economia; Facoemulsificação/economia

\section{INTRODUÇ̃̃̃O}

A cirurgia de extração de catarata tem se difundido em todo o mundo e realizada em sua grande maioria sob anestesia local ${ }^{(1)}$. Recentes estudos revelam que as técnicas anestésicas mais utilizadas incluem os métodos retrobulbar' peribulbar, subtenoniano e tópico ${ }^{(2)}$.

$\mathrm{Na}$ técnica retrobulbar a injeção do anestésico ocorre dentro do cone muscular por via transconjuntival ${ }^{(3-4)}$ ou percutânea ${ }^{(5-6)}$. São descritas complicações como hemorragia retrobulbar ${ }^{(5,7-9)}$, ptose pós-operatória permanente $^{(5)}$, trauma de nervo óptico ${ }^{(3,8-10)}$, perfuração de globo ocular ${ }^{(5,9-13)}$, disseminação do anestésico para o sistema nervoso central ${ }^{(3,5-6,8-10,14-16)}$, irritação meníngea ${ }^{(9)}$ e atrofia óptica ${ }^{(9,17)}$. Utilizando agulha entre 35 a 40 mm $^{(3-4,7,9)}$ de comprimento.

Muitas são as opções no que diz respeito ao tipo de anestésico desde sua descrição inicial em 1884 por Herman Knapp que utilizou solução aquosa de cocaína a $4 \%{ }^{(3)}$. Atualmente encontramos relatos do uso de mistura de bupivacaína $0,75 \%$ com lidocaína $2 \%{ }^{(3-4)}$ mistura de bupivacína $0,50 \%$ com lidocaína $2 \%{ }^{(3,7)}$ entre outros. Alguns autores adicionam à mistura anestésica hialuronidase ${ }^{(4,7)}$. Esta droga diminui o tempo de latência do anestésico por ajustar o pH local e melhora a sua difusão ${ }^{(6)}$. Pequenos 
52 Análise de custo dos materiais usados para realizar as técnicas anestésicas na cirurgia programada de extração de catarata por facoemulsificação

volumes do anestésico são rotineiramente usados $(2 \text { a } 5 \mathrm{ml})^{(4,7,9)}$ enfatizando que a injeção do material seja lenta ${ }^{(9)}$.

A anestesia peribulbar introduzida em 1970 por Kelmam ${ }^{(9)} \mathrm{e}$ primeiramente descrita em 1986 por Davis e Mandel ${ }^{(9)}$ apresentou-se como alternativa a anestesia retrobulbar, onde se injeta o agente anestésico no espaço extra-conal ${ }^{(18)}$. São relatadas complicações, embora raras ${ }^{(19)}$, como perfuração do globo ocular ${ }^{(10-11,20-23)}$, depressão de sistema nervoso central ${ }^{(10)}$, hemorragia periocular e retrobulbar $^{(20)}$, neuropatia óptica isquêmica aguda ${ }^{(10,20)}$, paralisia de musculatura extraocular ${ }^{(20-21)}$, aumento da pressão intra-ocu$\operatorname{lar}^{(20)}$ e anestesia e acinesia incompletas ${ }^{(21)}$. Encontraram-se inúmeras misturas de anestésicos como, bupivacaína $0,50 \%{ }^{(20)}$, bupivacaína $0,75 \%$ com lidocaína $1 \%{ }^{(5)}$. O volume de anestésico injetado varia na literatura entre 4 a $10 \mathrm{ml}^{(5,20)}$.

A anestesia local subtenoniana também chamada de anestesia "Flush"(2), ou método parabulbar ${ }^{(24-25)}$ é utilizada em cirurgias de catarata ${ }^{(26)}$, cirurgias filtrantes, vitrectomia ou cirurgias do segmento posterior ${ }^{(20)}$ e estrabismo ${ }^{(20,27)}$. Descrita por Stevens ${ }^{(28)}$ em 1992 e Greembaum ${ }^{(24)}$ no mesmo ano, consiste na administração via subtenoniana do anestésico no espaço retrobul$\operatorname{bar}^{(8,20,29)}$ com vantagem de injetar menor volume, levando pouco aumento da pressão posterior da órbita, não havendo necessidade de compressão orbitária para difusão da solução ${ }^{(30)}$. O tipo de anestésico pode ser bupivacaína $0,50 \%$ com lidocaína $2 \%{ }^{(20)}$, bupivacaína $0,75 \%$ com lidocaína $4 \%{ }^{(24)}$ entre outros. O volume injetado varia entre 2 a $5 \mathrm{ml}^{(8,20,24)}$.

A possibilidade de ocorrer perfuração ocular não foi relatada até os dias de hoje $\mathrm{e}^{(20)}$, mas a presença de hematoma retrobulbar foi descrita em um caso ${ }^{(31)}$, além de hemorragia subconjuntival e quemose ${ }^{(32)}$.

A anestesia tópica foi primeiramente descrita em $1884^{(32)}$ usando cocaína $5 \%$, porém não se tornou muito aceita devido a seus efeitos tóxicos ${ }^{(33)}$. Somente em 1991 Fichmam $^{(34-35)}$ utilizou tetracaína $0,5 \%$ em forma de colírio para realizar cirurgia de facoemulsificação sendo este utilizado até os dias de hoje para realizar cirurgias de catarata em córnea clara e túnel escleral, trabeculectomia $^{(36)}$ e estrabismo ${ }^{(37)}$. Kershner em 1993(32,35) usou no lugar da tetracaína a proparacaína $0,5 \%$ tópica para os mesmos fins. Recentemente o uso desta técnica vem aumentando como alternativa para reduzir os riscos associados com a injeção de anestésicos ${ }^{(38)}$. Este procedimento não leva a acinesia completa $^{(34,39)}$ bem como não bloqueia a inervação do corpo ciliar, podendo o paciente queixar-se de dor em procedimentos que levem a distorção ou pressão do olho ${ }^{(39)}$, principalmente em procedimentos sem sedação ${ }^{(32)}$.

Segundo Fukasaku ${ }^{(36)}$ uma técnica anestésica local ideal deveria satisfazer os seguintes critérios:

1. Ser segura - Para evitar possíveis traumas, os instrumentos introduzidos na órbita devem ser rombos; evitar a manipulação sem a direta observação das estruturas oculares evitando a manipulação às cegas, injeção de pouco volume de solução anestésica limitando os efeitos do aumento da pressão posterior da órbita e aumento da pressão intra-ocular.

2. Ser eficaz - Rápida, levando à completa anestesia de todas as estruturas oculares envolvidas no procedimento sem efeitos acinéticos e anestésicos desnecessários.
3. Levar a satisfação plena do paciente - O desconforto deve ser mínimo com poucos efeitos colaterais pós-operatórios.

No Brasil há escassez de estudos sobre custos em oftalmo$\operatorname{logia}^{(40)}$ tanto na área da propedêutica quanto na terapêutica clínica ou cirúrgica.

O objetivo deste estudo é comparar o custo das técnicas anestésicas locais em oftalmologia (retrobulbar, peribulbar, subtenoniana e tópica) utilizadas para realizar cirurgia de facoemulsificação.

\section{MÉTODOS}

Devido a grande variedade de descrições de técnicas anestésicas encontradas na literatura foram padronizadas, para a análise de custo dos materiais na realização dos diferentes procedimentos anestésicos, as técnicas anestésicas utilizadas no Setor de Catarata da Disciplina de Oftalmologia da Faculdade de Medicina do ABC. É rotina neste setor que o procedimento anestésico local seja realizado pelo próprio cirurgião, não sendo, portanto calculado o gasto com serviço de anestesiologia. Os pacientes são monitorizados por oximetria de pulso, eletrocardiografia, pressão sangüínea e pulsação. Apresentam ainda acesso a veia periférica para administração de possíveis drogas endovenosas.

Para cada técnica foram tabulados os valores totais correspondentes aos materiais necessários para realizar os procedimentos anestésicos em questão. Todos os materiais utilizados foram adquiridos após a análise de preços de três fornecedores, tendo sido escolhido o de menor valor.

O valor final de cada procedimento anestésico foi organizado em tabelas com valores de custo total e valores de custo para cada material. A seguir se encontram as descrições das técnicas anestésicas.

\section{Técnicas Anestésicas:}

1. Anestesia retrobulbar - Paciente posicionado em decúbito dorsal horizontal orientado a fixar os olhos em posição primária do olhar (ppo) após ter sido instilado duas gotas de colírio de tetracaína 0,5\%. A assepsia da pele de pálpebra superior e inferior feita com solução de Povidine tópico (PVPI) e gaze.

Realização da injeção na junção lateral do terço da rima orbitária inferior, utilizando seringa de $10 \mathrm{ml}$ e agulha de $30 \mathrm{~mm}$ de comprimento, ambas estéreis e descartáveis. A agulha avança posteriormente e logo depois do equador do globo é direcionado superiormente direto ao cone muscular, onde o anestésico é injetado A mistura anestésica contém 2,5 $\mathrm{ml}$ de bupivacaína $0,75 \%$ e $2,5 \mathrm{ml}$ de lidocaína $2 \%$ sem vasoconstritor (totalizando volume injetado de $5 \mathrm{ml})^{(41)}$.

O procedimento é realizado em sala anestésica separada e contígua ao centro cirúrgico, e o paciente é levado à mesa cirúrgica após ter completado todo o processo descrito anteriormente.

Os materiais e as quantidades necessárias ao procedimento estão no quadro 1 . 
2. Anestesia peribulbar - Paciente posicionado em decúbito dorsal horizontal orientado a fixar os olhos em posição primária do olhar (ppo), após ter sido instilado duas gotas de colírio de tetracaína $0,5 \%$. A assepsia da pele de pálpebra superior e inferior é feita com solução de Povidine tópico (PVPI) e gaze.

Realização do procedimento através do método de duas injeções. A primeira na junção do terço lateral com os dois terços medial da rima orbital inferior, através da pálpebra inferior, com direção cefálica e medial. A segunda na borda súpero-interna do globo ocular, através da pálpebra superior, utilizando seringa de $10 \mathrm{ml}$ e agulha de $30 \mathrm{~mm}$, ambas estéreis e descartáveis. A solução anestésica é preparada com Bupivacaína 0,75\% $20 \mathrm{ml}$ : retiram-se $6 \mathrm{ml}$, restando $14 \mathrm{ml}$ da solução. Dos $6 \mathrm{ml}$, despreza-se $1 \mathrm{ml}$ e com os restantes $5 \mathrm{ml}$ dissolve-se a hialuronidase (2000 UI), obtendo-se uma solução contendo $400 \mathrm{UI} / \mathrm{ml}^{-1}$. Misturam-se $2 \mathrm{ml}$ da solução de hialuronidase obtida (portanto $800 \mathrm{UI}$ ) com $14 \mathrm{ml}$ da solução original de bupivacaína, perfazendo um volume $16 \mathrm{ml}$ de solução do anestésico local contendo hialuronidase $50 \mathrm{UI} / \mathrm{ml}^{-1}$. Retiram-se $5 \mathrm{ml}$ dessa mistura e adiciona $5 \mathrm{ml}$ de lidocaína $2 \%$ sem vasoconstrictor ${ }^{(42)}$.

O volume injetado será de $5 \mathrm{ml}$ da solução do anestésico na primeira injeção e de $3 \mathrm{ml}$ na segunda (totalizando $8 \mathrm{ml}$ ), sendo depositado posterior ao equador do globo ocular em espaço periconal.

O procedimento é realizado em sala anestésica separada e contígua ao centro cirúrgico, e o paciente é levado à mesa cirúrgica após ter completado todo o processo descrito anteriormente.

Os materiais e as quantidades necessárias ao procedimento estão no quadro 2.

3. Anestesia subtenoniana - Paciente posicionado em decúbito dorsal horizontal orientado a olhar para cima, após ter sido instilado duas gotas de colírio de tetracaína $0,5 \%$. Realização de pequena incisão na conjuntiva e cápsula de Tenon a $3 \mathrm{~mm}$ do limbo no quadrante inferior nasal entre os músculos retos se for olho direito; e no quadrante inferior

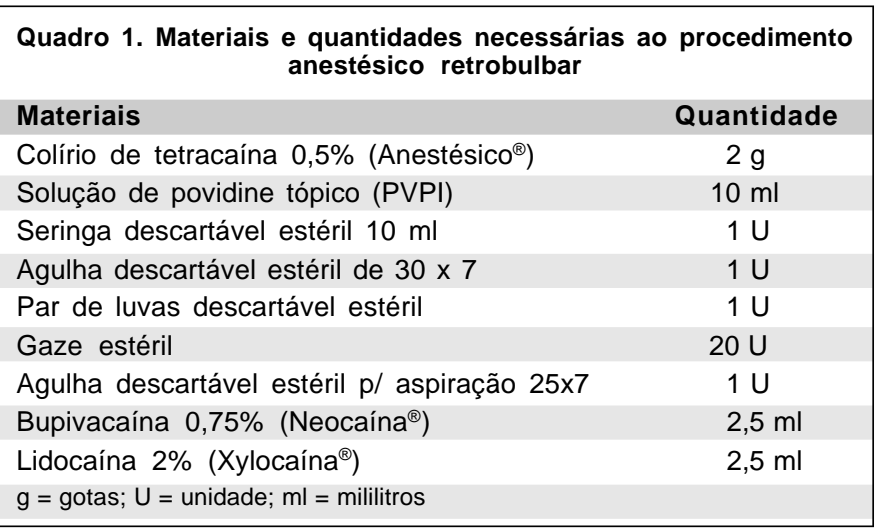

temporal se for olho esquerdo. O plano cirúrgico da ligação da Tenon com a esclera será dissecado.

Através do orifício no plano subtenoniano é introduzido uma cânula flexível de polietileno descartável, com ponta romba, chamada Cânula de Greenbaum acoplada a uma seringa de $3 \mathrm{ml}$ contendo mistura de $1 \mathrm{ml}$ de lidocaína a $2 \%$ com $1 \mathrm{ml}$ de bupivacaína $0,75 \%$. A solução anestésica será aplicada em bolo ("flush"). O anestésico será injetado rapidamente criando um jato de líquido que se espalha através dos espaços retro e parabulbar ${ }^{(24)}$.

O procedimento anestésico é realizado já com o paciente sobre a mesa cirúrgica, com os campos cirúrgicos montados para cirurgia, portanto a solução de PVPI, as gazes, e a luva cirúrgica foram as mesmas utilizadas para o resto da cirurgia.

Os materiais e as quantidades necessárias ao procedimento estão no quadro 3.

4. Anestesia tópica - A anestesia é realizada usando 2 gotas de colírio de tetracaína $0,5 \%$ instilada em fundo de saco inferior da conjuntiva ao entrar no centro cirúrgico, seguido de nova instilação da mesma substância após 3 minutos quando o paciente deita na maca cirúrgica e repetido mais uma vez após 2 minutos, exatamente no início da cirurgia ${ }^{(32)}$.

Os materiais e as quantidades necessárias ao procedimento estão no quadro 4.

\begin{tabular}{|c|c|}
\hline Materiais & Quantidade \\
\hline Colírio de tetracaína $0,5 \%\left(\right.$ Anestésico $\left.{ }^{\circledR}\right)$ & $2 \mathrm{~g}$ \\
\hline Solução de povidine tópico (PVPI) & $10 \mathrm{ml}$ \\
\hline Seringa descartável estéril $10 \mathrm{ml}$ & $1 \mathrm{U}$ \\
\hline Agulha descartável estéril de $30 \times 7$ & $1 \mathrm{U}$ \\
\hline Par de luvas descartável estéril & $1 U$ \\
\hline Gaze estéril & $20 \mathrm{U}$ \\
\hline Agulha descartável estéril p/ aspiração $25 \times 7$ & $1 U$ \\
\hline Bupivacaína $0,75 \%$ (Neocaína $^{\circledR}$ ) & $6,6 \mathrm{ml}$ \\
\hline Lidocaína 2\% (Xylocaína $^{\circledR}$ ) & $5 \mathrm{ml}$ \\
\hline Hialuronidase 2000 UI $\left(\right.$ Hialozima $\left.^{\circledR}\right)$ & $1 \mathrm{amp}$ \\
\hline
\end{tabular}

Quadro 3. Materiais e quantidades necessários ao procedimento anestésico subtenoniano

\section{Materiais}

Quantidade

Colírio de tetracaína $0,5 \%$ (Anestésico ${ }^{\circledR}$ )

$2 \mathrm{~g}$

Seringa descartável estéril $5 \mathrm{ml}$

$1 \mathrm{U}$

Agulha descartável estéril $\mathrm{p}$ / aspiração $25 \times 7$

$1 \mathrm{U}$

Bupivacaína $0,75 \%$ (Neocaína ${ }^{\circledR}$ )

Lidocaína 2\% (Xylocaína $^{\circledR}$ )

$1 \mathrm{ml}$

Cânula de Greenbaum (Alcon ${ }^{\circledR}$ )

$1 \mathrm{ml}$

$1 \mathrm{U}$

$\mathrm{g}$ = gotas; $\mathrm{U}=$ unidade; $\mathrm{ml}=$ mililitros 
54 Análise de custo dos materiais usados para realizar as técnicas anestésicas na cirurgia programada de extração de catarata por facoemulsificação

\begin{tabular}{l}
$\begin{array}{l}\text { Quadro 4. Materiais e quantidades necessários ao procedimento } \\
\text { anestésico tópico }\end{array}$ \\
$\begin{array}{l}\text { Materiais } \\
\text { Colírio de tetracaína } 0,5 \% \text { (Anestésico }^{\circledR} \text { Quantidade } \\
\mathrm{g}=\text { gotas }\end{array}$ \\
\hline
\end{tabular}

\section{RESULTADOS}

Os resultados a seguir se referem aos menores valores encontrados após a pesquisa entre três representantes de insumos hospitalares. Estão organizados em tabelas específicas para cada procedimento.

$\mathrm{Na}$ tabela 1 encontra-se o valor do custo da cada material em suas respectivas quantidades, bem como o custo total para realizar a anestesia retrobulbar (Tabela 1).

Na tabela 2 pode-se observar o valor do custo da cada material em suas respectivas quantidades, bem como o custo total para realizar a anestesia peribulbar (Tabela 2).

Na tabela 3 observar-se o valor do custo da cada material em suas respectivas quantidades, bem como o custo total para realizar a anestesia subtenoniana (Tabela 3).

Na tabela 4 pode-se observar o valor do custo da cada material em suas respectivas quantidades, bem como o custo total para realizar a anestesia tópica (Tabela 4).

O custo das anestesias e o valor médio gasto com cada material para realizar cada procedimento anestésico encontra-se na tabela 5 .

\section{DISCUSSÃO}

A procura pelo método anestésico ideal representa ainda um grande desafio para os cirurgiões oftalmológicos ${ }^{(2)}$. A técnica anestésica deve apresentar mínimos riscos ao paciente ${ }^{(43)}$, maior segurança para o médico ${ }^{(43)}$ e ser pouco onerosa ao sistema de saúde, tanto público quanto privado. Evidentemente apenas o custo não é suficiente para a decisão de qual técnica anestésica deverá ser utilizada, existem questões que precisam ser avaliadas ${ }^{(9)}$ como: a técnica escolhida deverá proporcionar anestesia e acinesia satisfatórias ${ }^{(8,20)}$, ser de fácil realização $^{(9)}$ e segura ${ }^{(5)}$.

Tende-se a escolher como método mais viável a anestesia tópica uma vez que o seu custo é bastante baixo quando se analisa apenas o custo final de cada procedimento,

Seu custo foi de 0,06 Reais, significativamente menor do que a da anestesia peribulbar, subtenoniana e retrobulbar, porém não leva a acinesia e não há anestesia de todo globo ocular, pois não há ação do agente anestésico sobre o gânglio ciliar devendo o tempo cirúrgico ser o menor possível ${ }^{(39)}$. A ação da tetracaína $1 \%$ tópica tem inicio entre 13 e 30 segundos e duração de 15 a 20 minutos após a instilação ${ }^{(44)}$ o que dificulta o procedimento cirúrgico para oftalmologistas menos experientes que não estejam aptos a trabalhar com um olho sem acinesia $^{(45)}$ ou ainda em pacientes pouco colaborativos mesmo em mãos extremamente hábeis ${ }^{(34)}$. Porém o desenvolvimento de novas incisões, aparelhos e instrumentais de facoemulsificação associados a modernas tecnologias de materiais de lentes intra-oculares e substâncias viscoelásticas vêm tornando a anestesia tópica cada vez mais difundida ${ }^{(45-46)}$, principalmente pela rápida recuperação visual do paciente ${ }^{(45)}$. A anestesia tópica associada à injeção de anestésico intra-camerular vem ganhando maior aceitação no meio oftalmológico. Vários trabalhos vêm sendo realizados com esta técnica e sugerem diminuição da escala de dor e sensação de desconforto por parte do paciente. Contudo esta associação também não leva a acinesia, portanto exige boa experiência do cirurgião em facoemulsificação além de tornar o método mais oneroso ${ }^{(45)}$.

A anestesia retrobulbar tornou-se o método de escolha para grande maioria dos cirurgiões oftalmológicos ${ }^{(47-48)}$. Considerada de baixo custo, portanto percebe-se que ela apresenta grande associação com complicações severas, como hemorragias, perfuração do globo ocular e disseminação para o sistema nervoso central, entre outras. Por outro lado garante ao paciente acinesia e anestesia satisfatória traduzindo ao médico segurança no ato cirúrgico. A anestesia é realizada utilizando parâmetros e marcas anatômicas externas e, portanto a

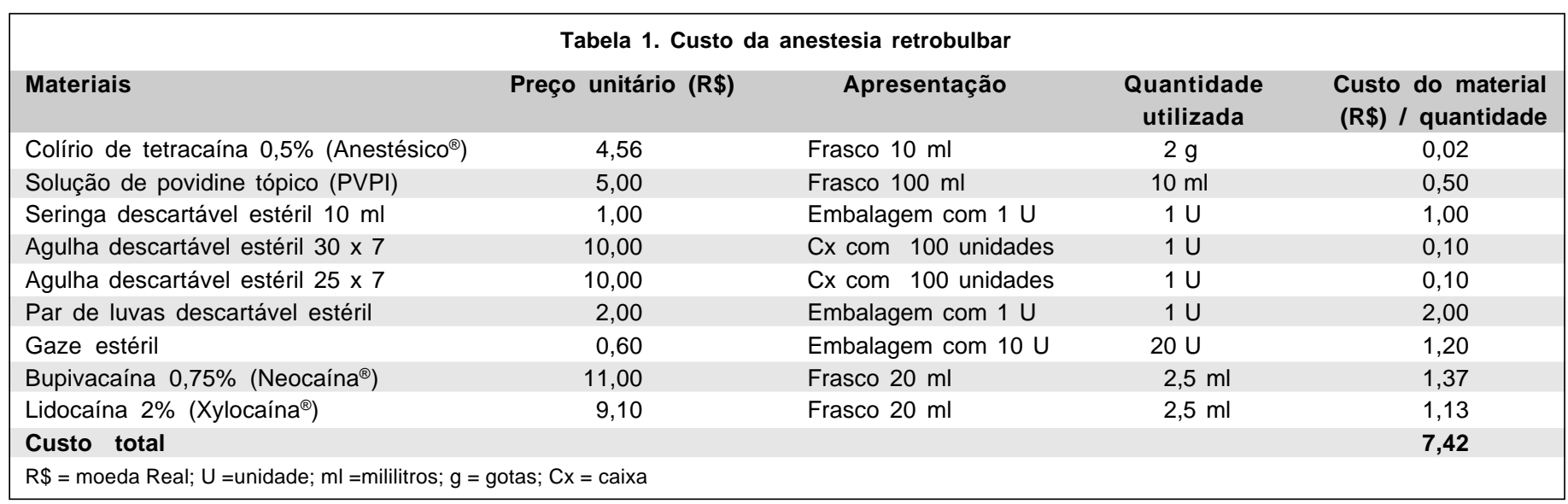




\begin{tabular}{|c|c|c|c|c|}
\hline Materiais & Preço unitário $(\mathbf{R} \$)$ & Apresentação & $\begin{array}{c}\text { Quantidade } \\
\text { utilizada }\end{array}$ & $\begin{array}{l}\text { Custo do material } \\
\text { (R\$) / quantidade }\end{array}$ \\
\hline Colírio de tetracaína $0,5 \%\left(\right.$ Anestésico $\left.{ }^{\circledR}\right)$ & 4,56 & Frasco $10 \mathrm{ml}$ & $2 \mathrm{~g}$ & 0,02 \\
\hline Solução de povidine tópico (PVPI) & 5,00 & Frasco $100 \mathrm{ml}$ & $10 \mathrm{ml}$ & 0,50 \\
\hline Seringa descartável estéril $10 \mathrm{ml}$ & 1,00 & Embalagem com $1 \mathrm{U}$ & $1 U$ & 1,00 \\
\hline Agulha descartável estéril $30 \times 7$ & 10,00 & Cx com 100 unidades & $1 U$ & 0,10 \\
\hline Agulha descartável estéril $25 \times 7$ & 10,00 & Cx com 100 unidades & $1 \mathrm{U}$ & 0,10 \\
\hline Par de luvas descartável estéril & 2,00 & Embalagem com $1 \mathrm{U}$ & $1 U$ & 2,00 \\
\hline Gaze estéril & 0,60 & Embalagem com $10 \mathrm{U}$ & $20 \mathrm{U}$ & 1,20 \\
\hline Bupivacaína $0,75 \% \quad\left(\right.$ Neocaína $\left.^{\circledR}\right)$ & 11,00 & Frasco $20 \mathrm{ml}$ & $6,6 \mathrm{ml}$ & 3,63 \\
\hline Lidocaína 2\% (Xylocaína $^{\circledR}$ ) & 9,10 & Frasco $20 \mathrm{ml}$ & $5 \mathrm{ml}$ & 2,25 \\
\hline Hialuronidase 2000 UI (Hialozima $^{\circledR}$ ) & 42,50 & Embalagem c/ 3 amp & $1 \mathrm{amp}$ & 14,16 \\
\hline Custo total & & & & 24,96 \\
\hline
\end{tabular}

\begin{tabular}{|c|c|c|c|c|}
\hline Materiais & Preço unitário $(\mathbf{R} \$)$ & Apresentação & $\begin{array}{c}\text { Quantidade } \\
\text { utilizada }\end{array}$ & $\begin{array}{l}\text { Custo do material } \\
\text { (R\$) / quantidade }\end{array}$ \\
\hline Colírio de tetracaína $0,5 \%$ (Anestésico ${ }^{\circledR}$ ) & 4,56 & Frasco $10 \mathrm{ml}$ & $2 \mathrm{~g}$ & 0,02 \\
\hline Seringa descartável estéril $5 \mathrm{ml}$ & 1,00 & Embalagem $1 \mathrm{U}$ & $1 \mathrm{U}$ & 1,00 \\
\hline Agulha descartável estéril $25 \times 7$ & 10,00 & Cx com 100 unidades & $1 U$ & 0,10 \\
\hline Bupivacaína $0,75 \% \quad\left(\right.$ Neocaína $\left.^{\circledR}\right)$ & 11,00 & Frasco $20 \mathrm{ml}$ & $1 \mathrm{ml}$ & 0,55 \\
\hline Lidocaína 2\% (Xylocaína $^{\circledR}$ ) & 9,10 & Frasco $20 \mathrm{ml}$ & $1 \mathrm{ml}$ & 0,45 \\
\hline Cânula de Greenbaum (Alcon $\left.{ }^{\circledR}\right)$ & 46,00 & Embalagem $1 \mathrm{U}$ & $1 \mathrm{U}$ & 46,00 \\
\hline Custo total & & & & 48,12 \\
\hline
\end{tabular}

\begin{tabular}{|lccc|}
\hline & \multicolumn{2}{c|}{ Tabela 4. Custo da anestesia tópica } & \\
Materiais & Preço unitário (R\$) & $\begin{array}{c}\text { Apresentação } \\
\text { Quantidade } \\
\text { utilizada }\end{array}$ & $\begin{array}{c}\text { Custo do material } \\
\text { (R\$) / quantidade }\end{array}$ \\
$\begin{array}{l}\text { Colírio de tetracaína } 0,5 \%\left(\text { Anestésico }{ }^{\boxplus}\right) \\
\text { Custo total }\end{array}$ & 4,56 & Frasco $10 \mathrm{ml}$ & 0,06 \\
$\mathrm{R} \$=$ moeda Real; $\mathrm{ml}=$ mililitros; $\mathrm{g}=$ gotas & & & $\mathbf{0 , 0 6}$ \\
\hline
\end{tabular}

\begin{tabular}{|lccc|}
\hline \multicolumn{3}{|l|}{ Tabela 5. Custo das anestesias e gasto médio de cada material } \\
Anestesia & Custo (R\$) & $\begin{array}{c}\text { Quantidade } \\
\text { de material }\end{array}$ & $\begin{array}{c}\text { Gasto médio } \\
\text { com cada material }\end{array}$ \\
Tópica & 0,06 & 1 & 0,06 \\
Retrobulbar & 7,42 & 9 & 0,82 \\
Peribulbar & 24,96 & 10 & 2,49 \\
Subtenoniana & 48,12 & 6 & 8,02 \\
$R \$=$ moeda Real & & & \\
\hline
\end{tabular}

utilização de agulhas com bisel rombo ${ }^{(8,11,45)}$ e com a face voltada para o globo ${ }^{(11,45)}$, administração lenta do anestésico e observação do nível de consciência do paciente reduzem as chances de acidentes ${ }^{(45)}$. Suzuki e colaboradores em estudo comparativo com a anestesia tópica relataram predisposição para diminuição da pressão sanguínea nos pacientes que utilizaram o bloqueio retrobulbar, mas as razões para este fenômeno ainda não são claras $^{(49)}$.

A anestesia peribulbar surgiu como alternativa a retrobulbar apresentando a mesma eficiência (acinesia e analgesia), porém com maior segurança, uma vez que a injeção do anestésico ocorre fora do cone muscular e ainda a agulha está longe da parede do globo ocular, nervo óptico e da bainha dural ${ }^{(5)}$. Todavia o volume de anestésico utilizado é maior quando comparado ao da retrobulbar, tornando o procedimento mais oneroso. Calculando apenas o custo do anestésico gasto em cada um dos procedimentos anteriores, notamos que para a peribulbar gastamos 5,88 Reais, pois utilizamos 6,6 ml de bupivacaína $0,75 \%$ e $5 \mathrm{ml}$ de lidocaína $2 \%$ e para a retrobulbar utilizando 2,5 $\mathrm{ml}$ de bupivacaína $0,75 \%$ e 2,5 $\mathrm{ml}$ de lidocaína $2 \%$ foi gasto 2,50 Reais. A apresentação de cada frasco, tanto da 
56 Análise de custo dos materiais usados para realizar as técnicas anestésicas na cirurgia programada de extração de catarata por facoemulsificação

bupivacaína $0,75 \%$ quanto da lidocaína $2 \%$ é de $20 \mathrm{ml}$, isto significa que se consegue realizar 8 bloqueios retrobulbares com 1 frasco de bupivacaína $0,75 \%$ e 8 com o da lidocaína $2 \%$ e apenas 3 bloqueios peribulbar com 1 frasco de bupivacaína $0,75 \%$ e 4 com o da lidocaína $2 \%$.

O uso da hialuronidase apresenta também um aumento do custo na realização da anestesia peribulbar. Esta droga é responsável por $61,35 \%$ do valor total do procedimento em questão.

Estudos revisados na literatura sobre a utilização da hialuronidase mostram que o seu uso para realização da anestesia peribulbar ainda é conflitante ${ }^{(46)}$. Damasceno e colaboradores apresentaram bons resultados quando não utilizaram a hialuronidase, pois acham que por um lado essa droga acelera o tempo de início da ação do anestésico ${ }^{(4,6)}$, mas por outro aumenta a permeabilidade dos tecidos adjacentes ${ }^{(4,6,46)}$, fazendo com que o tempo de ação do anestésico fique diminuído, e ainda aumenta o volume da solução injetada na cavidade orbitária ${ }^{(4)}$. Optando por não usá-la o custo dessa técnica cairia para 10,80 reais, valor próximo ao da retrobulbar, mas com as vantagens de ser um procedimento mais seguro ${ }^{(41)} \mathrm{com}$ menor risco de perfuração ocular, hemorragia, toxicidade e ainda causar menor aumento da pressão intra-orbitária.

A anestesia subtenoniana surge como um método bastante eficaz, apresentando bom controle da dor ${ }^{(50)}$ tendo como característica injetar pequena quantidade $(2 \mathrm{ml})$ de anestésico diretamente no cone muscular, diferentemente da anestesia retrobulbar na qual o local da injeção é o mesmo, mas o volume injetado é maior $(5 \mathrm{ml})$. Devido ao fato do anestésico agir diretamente sobre o gânglio ciliar a obtenção de boa acinesia e anestesia está favorecida.

As complicações observadas com essa técnica como quemose, hemorragia subconjuntival e risco de lesão das veias vorticosas são pouco freqüentes e os riscos de perda ou déficit visual permanente são muito $\operatorname{raros}^{(45)}$. Jaycock e colaboradores descreveram 3 casos de paralisia de reto inferior após a injeção no espaço subtenoniano, mas com efeito reversível ${ }^{(51)}$. Portanto as complicações das injeções às cegas como é o caso das técnicas retrobulbar e peribulbar são evitadas e ainda mostrando evidências, em estudo comparativo, da administração da injeção via subtenoniana ser menos dolorosa que a retrobulbar ${ }^{(50)}$.

A desvantagem desta técnica é o custo bastante elevado $(48,12$ reais) quando comparada a retrobulbar (7,42 reais), peribulbar (24,96 reais) e tópica ( 0,06 reais). Apesar do número de materiais gastos na subtenoniana ser pequeno, o que a torna cara é a utilização da cânula de Greenbaum que isolada tem valor de 46,00 reais ou seja $95,5 \%$ do valor total do procedimento. A cânula tem como característica ser redonda na superfície superior e achatada na superfície posterior, o que facilita a introdução embaixo da Tenon.

Talvez uma opção para torná-la menos onerosa seria usar outra cânula rígida e romba. Esta opção é mais barata, apresenta os mesmos resultados da primeira tendo a diferença de ser rígida. Portanto o cirurgião necessitará de adaptação ao uso dessa cânula para realizar o procedimento já seu conhecido, porém o custo do procedimento seria 17,12 reais, ainda mais caro quando comparada a retrobulbar e a tópica, mas, podendo ser utilizada em pacientes pouco colaborativos e por um maior número de cirurgiões, desde os iniciantes até os mais experientes.

\section{CONCLUSÕES}

A análise de custos dos materiais necessários para a realização das técnicas anestésicas na facoemulsificação, realizadas pelo cirurgião oftalmológico, no Setor de Catarata da Faculdade de Medicina da Fundação ABC apresentou as seguintes conclusões:

1. A anestesia tópica foi a técnica que apresentou o menor custo.

2. A anestesia retrobulbar apresenta um custo mais baixo quando comparado à peribulbar e subtenoniana.

3. A anestesia subtenoniana apresenta um custo mais elevado quando comparada a outras técnicas utilizadas neste estudo.

\section{ABSTRACT}

Purpose: To compare the costs of local anaesthesia (retrobulbar block, peribulbar block, sub-Tenon and topical) to perform phacoemulsification cataract extraction. Methods: We studied the costs of the materials that are necessary to perform the local anaesthesia (retrobulbar block, peribulbar block, sub-Tenon and topical). We adopted the standard technique used for the Cataract Sector of the Department of Ophthalmology, ABC University. For each technique the total price of the each item to perform the above described local anaesthesia was analyzed. Prices were obtained from three different stores which sell hospitals materials and we considered for this study the item with a lower price. Results: The costs of topical anaesthesia were 0.06 reais, for the retrobulbar it was 7.42 reais, 24.96 reais for the peribulbar was and 48.12 reais for the sub-Tenon. Conclusions: 1. Topical anaesthesia showed the lowest cost. 2. Retrobulbar anaesthesia showed low cost when compared with the peribulbar and sub-Tenon 3. Sub-Tenon's anaesthesia showed the highest cost.

Keywords: Health care costs; Anesthesia, local/economy; Cataract extraction/economy; Phacoemulsification/economy

\section{REFERÊNCIAS}

1. Katayama M, Silva SMM, Vieira JL, Arrigucci RJ. Estudo clínico comparativo de duas técnicas de bloqueio peribulbar para cirurgia oftálmica. Rev Bras Anestesiol 1993;43:167-74.

2. Boyd BF. Progressos na anestesia local. Highlights Ophthalmol, 1995;23: 56-64 [Edição brasileira].

3. Feibel RM. Current concepts in retrobulbar anesthesia. Surv Ophthalmol 1985;30:102-10.

4. Damasceno EF, Caldas JAS, Mello MZ, Martin RHS, Encina RAM. Anestesia peribulbar e retrobulbar: análise comparativa. Rev Bras Oftalmol 1990;49:27-33. 
5. Davis DB 2 $2^{\text {nd }}$, Mandel MR. Anesthesia for cataract extraction. Int Ophthalmol Clin 1994;34:13-30.

6. Nicoll JM, Treuren B, Acharya PA, Ahlen K, James M. Retrobulbar anesthesia: the role of hyaluronidase. Anesth Analg 1986;65:1324-8.

7. Edge KR, Nicoll JM. Retrobulbar hemorrhage after 12,500 retrobulbar blocks. Anesth Analg 1993;76:1019-22.

8. Bergman L, Berglin L, Algvere PV, Laurell CG, Stenkula S. Limbal subTenon's administration of retrobulbar anesthesia using a blunt irrigating cannula. Ophthalmic Surg Lasers 1996;27:106-12.

9. Davis DB 2nd, Mandel MR. Posterior peribulbar anaesthesia: an alternative to retrobulbar anaesthesia. J Cataract Refract Surg 1986;12:182-4.

10. Berglin L, Stenkula S, Agvere PV. Ocular perforation during retrobulbar and peribulbar injections. Ophthalmic Surg Lasers 1995;26:429-34.

11. Birch AA, Evans M, Redembo E. The ultrasonic localization of retrobulbar needles during retrobulbar block. Ophthalmology 1995;102:824-6.

12. Hamilton RC. Ocular explosion after peribulbar anesthesia. Ophthalmology 1998;105:1355.

13. Mc Combe M, Heriot W. Penetrating ocular injury following local anaesthesia. Aust N Z J Ophthalmol 1995;23:33-6.

14. Nicoll JM, Acharya PA, Ahlen K, Baguneid S, Edge KR. Central nervous system complications after 6,000 retrobulbar blocks. Anesth Analg 1987; 66:1298-302.

15. Wittpenn JR, Rapoza P, Sternberg P Jr, Kuwashima L, Saklad J, Patz A. Respiratory arrest following retrobulbar anaesthesia. Ophthalmology 1986; 93:867-70.

16. Ribeiro RMS. Apnéia pós-bloqueio peribulbar em cirurgia de catarata: relato de caso e revisão da bibliografia. Arq Bras Oftalmol 1999;62:262-4.

17. Carroll FD. Optic nerve complications of cataract extraction. Trans Am Acad Ophthalmol Otolaryngol. 1973;77:OP623-9.

18. Rubin AP. Local anaesthesia for ophthalmic surgery: an anaesthetist's view. Eur J Implant Refract Surg 1993;5:8-11.

19. Roman SJ, Chong Sit DA, Boureau CM, Auclin FX, Ullern MM. SubTenon's anaesthesia: an efficient and safe technique. Br J Ophthalmol 1997; 81:673-6.

20. Azmon B, Alster Y, Lazar M, Geyer O. Effectiveness of sub-Tenon's versus peribulbar anesthesia in extracapsular cataract surgery. J Cataract Refract Surg 1999;25:1646-50.

21. Davis DB 2nd, Mandel MR. Efficacy and complication rate of 16,224 consecutive peribulbar blocks. A prospective multicenter study. J Cataract Refract Surg 1994;20:327-37.

22. Duker JS, Belmont JB, Benson WE, Brooks HL Jr, Brown GC, Federman $\mathrm{JL}$ et al. Inadvertent globe perforation during retrobulbar and peribulbar anesthesia. Patient characteristics, surgical management, and visual outcome. Ophthalmology 1991;98:519-26.

23. Gillow JT, Aggarwal RK, Kirkby GR. Ocular perforation during peribulbar anaesthesia. Eye 1996;10:533-6.

24. Greenbaum S. Parabulbar anesthesia. Am J Ophthalmol 1992;114:776

25. Schargel K. Anestesia parabulbar en procedimentos quirúrgicos oftalmológicos [Tese]. Caracas: Universidad Central de Venezuela, Faculdad de Medicina, 1997.

26. Hansen EA, Mein CE, Mazzoli R. Ocular anesthesia for cataract surgery: a direct sub-Tenon's approach. Ophthalmic Surg 1990;21:696-9.

27. Fujishima H, Yagi Y, Yang HY, Toda I, Shimazaki J, Tsubota K. Direct sub-Tenon's ocular anesthesia for strabismus surgery. Ophthalmologica 1995;209:208-11.

28. Stevens JD. A new local anesthesia technique for cataract extraction by one quadrant sub Tenon's infiltration. Br J Ophthalmol 1992;76:670-4.
29. Bak A, Marczak M. Znieczulenie przygalkowe kaniula Greenbauma do najczesciej wykonywanych operacji okulistycznych. Klin Oczna 1999;101: 367-70.

30. Verghese I, Sivaraj R, Lai YK. The effectiveness of sub-Tenon's infiltration of local anaesthesia for cataract surgery. Aust N Z J Ophthalmol 1996;24:117-20.

31. Olitsky SE, Juneja RG. Orbital hemorrhage after the administration of subTenon's infusion anesthesia. Ophthalmic Surg Lasers 1997;28:145-6.

32. Zafirakis P, Voudouri A, Rowe S, Livir-Rallatos G, Livir-Rallaton C, Canakis C et al. Topical versus sub-Tenon's anesthesia without sedation in cataract surgery. J Cataract Refract Surg 2001;27:873-9.

33. Vale, MB, Garrido MF, Gonçalves VBD, Cunha RN. Irrigação sub-tenoneana em cirurgia vitreo-retiniana. Rev Bras Oftalmol 1999;58:295-8.

34. Bernardes F, Dias FR. Anestesia tópica em cirurgia de catarata. In: Dias FR Cirurgia da catarata: Rio de Janeiro:Cultura Médica; 2000. p. 49-52.

35. Lu LW. Selección y preparación del paciente. Anestesia. In: Padilha MA, Argento C, Lu LW, editors. Facoemulsificacion de lo básico a lo avanzado. Rio de Janeiro: RioMed Livros; 1996. p.35-56.

36. Fukasaku H, Marron JA. Pinpoint anesthesia: a new approach to local ocular anesthesia. J Cataract Refract Surg 1994;20:468-71.

37. Klyve P, Nicolaissen B Jr. Topical anesthesia and adjustable sutures in strabismus surgery. Acta Ophthalmol 1992;70:637-40.

38. Katz J, Feldman MA, Bass EB, Lubomski LH, Tielsch JM, Petty BG et al. Injectable versus topical anesthesia for cataract surgery: patient perceptions of pain and side effects. The Study of Medical Testing for Cataract Surgery study team. Ophthalmology 2000;107:2054-60.

39. Jolliffe DM, Abdel-Khalek MN, Norton AC. A comparison of topical anaesthesia and retrobulbar block for cataract surgery. Eye 1997;11:858-62.

40. Pedroso L, Carvalho Junior ES, Paranhos Junior A, Prata Junior JA, Mello PAA. Custo real do tratamento do glaucoma para o paciente. Arq Bras Oftalmol 1999;62:677-82.

41. Loots JH, Venter JA. Posterior peribulbar anaesthesia for intra-ocular surgery. S Afr Med J 1988;74:507-9.

42. Serzedo PSM, Nociti JR, Zuccolotto EB, Cagnolati CA, Nunes AMM Ropivacaína em bloqueio peribulbar: estudo comparativo com bupivacaína. Rev bras anestesiol 1998;48:258-63.

43. Briggs MC, Beck SA, Easakowitz L. Sub-Tenon's versus peribulbar anaesthesia for cataract surgery. Eye 1997;11:639-43.

44. Bartlett JD, Fiscella RG, Benett E, Jannus SD, Rowsey JJ, et al. Local anesthetics. In: Ophthalmic drugs facts, St Louis: Facts and Comparisons; 2000. p.3-35.

45. Brisola IV, Shiroma HF. Anestesia para cirurgia de catarata. In: Arieta CE editor. Manual do CBO cristalino e catarata 2002. Rio de Janeiro: Cultura Médica; 2002. p.73-92.

46. Rowley SA, Hale JE, Finlay RD. Sub-Tenon's local anaesthesia: the effect of hyaluronidase. Br J Ophthalmol 2000;84:435-6.

47. Weiss JL, Deichman CB. A comparison of retrobulbar and periocular anesthesia for cataract surgery. Arch Ophthalmol 1989;107:96-8.

48. Sanders R, Ahmed S, Craig EW, Young JD. Comparison of catecholamine and pressor effects in peribulbar and retrobulbar anaesthesia in cataract surgery. Eye 1997;11:644-8.

49. Suzuki R, Kuroki S, Fujiwara N. A comparison of blood pressure changes in phacoemulsification cataract surgery with topical and retrobulbar block local anesthesia. Graefes Arch Clin Exp Ophthalmol 1997;235:277-82.

50. Friedman DS, Bass EB, Lubomski LH, Fleisher LA, Kempen JH, Magaziner $\mathrm{J}$ et al. Synthesis of the literature on the effectiveness of regional anesthesia for cataract surgery. Ophthalmology 2001;108:519-29.

51. Jaycock PD, Mather CM, Ferris JD, Kirkpatrick JN. Rectus muscle trauma complicating sub-Tenon's local anaesthesia. Eye 2001; 15:583-6.

\section{Ao enviar um artigo para publicação,} leia ATENTAMENTE as instruções para autores, constante no final de cada fascículo. 